\title{
Using Lyve1-Cre+ Mice to Visualize Lymphatics for Laser Capture Microdissection
}

\author{
Aadarsh Patel ${ }^{1}$, Ganesh Mohan ${ }^{1}$, Imran Khan ${ }^{1}$, Mithun Sinha ${ }^{1}$, Aladdin H. Hassanein ${ }^{1}$ \\ ${ }^{1}$ Department of Surgery, Indiana Center for Regenerative Medicine and Engineering, Indiana \\ University School of Medicine
}

\begin{abstract}
Background:
Lymphedema is characterized by limb swelling secondary to lymphatic dysfunction. Lymphedema most frequently develops following breast cancer treatment due to iatrogenic damage of the lymphatics from surgery and radiation. Lymphedema affects $20-40 \%$ of breast cancer survivors. There is no cure for this disease. For determination of successful delivery of gene-based therapies, target cells are often isolated and analyzed via real-time PCR. One method to isolate a region of cells within a tissue section is laser capture microdissection (LCM). This process involves outlining the desired regions, which are cut on membrane slides and captured using a laser. Using LCM requires visualization and identification of the target tissue. In the case of lymphedema therapies, the target tissue is lymphatic vasculature.
\end{abstract}

\section{Rationale of Project:}

While lymphatics can be visualized using immunohistochemistry antibodies specific to lymphatic markers, the process is time consuming and can interfere with RNA levels in the tissue. Another option to visualize lymphatics is to use Lyve1-Cre ${ }^{+}$mice. These mice express enhanced green fluorescent protein on lymphatic cells. The purpose of this project is to assess the utility of Lyve1$\mathrm{Cre}^{+}$and develop the methodology to capture the lymphatics in the murine tail to enable utilization of this methodology in murine tail model of lymphedema.

\section{Methodology Development:}

Samples of Lyve1-Cre ${ }^{+}$mouse tails were harvested. Sections $(10 \mu \mathrm{m})$ were captured on LCM slides and dehydrated. The slides were visualized on the LCM microscope system, lymphatics vessels fluoresced green. They were captured via laser dissection. The captured samples were analyzed for the lymphatic specific genes (Prox1 and Lyve1) via quantitative real-time PCR to determine the purity of capture. 